\title{
ORIGINAL ARTICLE Downregulation of CXCL12 in mesenchymal stromal cells by TGF $\beta$ promotes breast cancer metastasis
}

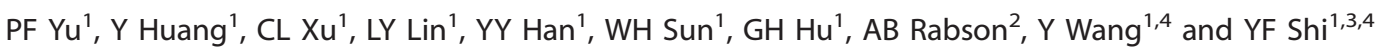

\begin{abstract}
Mesenchymal stromal cells (MSCs) are one of major components of the tumour microenvironment. Recent studies have shown that MSC tumour residence and their close interactions with inflammatory factors are important factors that affect tumour progression. Among tumour-associated inflammatory factors, transforming growth factor $\beta$ (TGF $\beta$ ) is regarded as a key determinant of malignancy. By employing a lung metastasis model of a murine breast cancer, we show here that the prometastatic effect of MSCs was dependent on their response to TGF $\beta$. Interestingly, we found that MSC-produced CXCL12, an important chemokine in tumour metastasis, was markedly inhibited by TGF $\beta$. Furthermore, silencing of CXCL12 in TGF $\beta$-unresponsive MSCs restored their ability to promote tumour metastasis. We found that 4T1 breast cancer cells expressed high levels of CXCR7, but not of CXCR4, both of which are CXCL12 receptors. In presence of CXCL12, CXCR7 expression on tumour cells was decreased. Indeed, when CXCR7 was silenced in breast cancer cells, their metastatic ability was inhibited. Therefore, our data demonstrated that sustained expression of CXCL12 by MSCs in the primary tumour site inhibits metastasis through reduction of CXCR7, while, in the presence of TGF $\beta$, this CXCL12 effect of MSCs on tumour cells is relieved. Importantly, elevated CXCR7 and depressed CXCL12 expression levels were prominent features of clinical breast cancer lesions and were related significantly with poor survival. Our findings reveal a novel mechanism of MSC effects on malignant cells through which crosstalk between MSCs and TGF $\beta$ regulates tumour metastasis.
\end{abstract}

Oncogene (2017) 36, 840-849; doi:10.1038/onc.2016.252; published online 26 September 2016

\section{INTRODUCTION}

Studies of the interactions between tumour cells and the tumour stroma in regulating tumour metastasis have been the subject of intense investigations. Many types of cells, such as immune cells, endothelial progenitor cells and mesenchymal stromal cells (MSCs), are recruited to the stroma of developing tumours. ${ }^{1-4}$ MSCs are pluripotent stem cells, and are well known for their ability to maintain tissue homeostasis and regenerate the damaged tissue by sensing signals released from the injured tissue..$^{5-7}$ These mechanisms also appear to operate in tumours, consistent with the concept that a tumour resembles a wound that does not heal. ${ }^{8}$ Experimental evidence has shown that the direct effects of MSCs on tumour cells can enhance tumour metastasis. ${ }^{9,10}$ Thus, the metastatic phenotype of tumour cells is not only dependent on autonomous changes in tumour cells, ${ }^{11-13}$ but is also influenced by tumour stroma cells in tumour microenvironment. The complexity of MSCs in regulating different types of tumour development, such as breast cancer, was associated with their ability to secrete plentiful growth factors that support tumour growth and angiogenesis, as well as their role in moulding the tumour immune microenvironment by facilitating monocyte and macrophage infiltration, and suppressing antitumour T-cell activity. ${ }^{14-16}$ However, the functions of MSCs in breast cancer metastasis have not been fully elucidated.

Investigations on MSCs within the stroma of breast cancer xenografts showed that MSCs can enhance the xenograft metastatic ability to the lungs and the bones. The contributions of MSCs to breast cancer metastasis are mostly mediated through their ability to produce a number of factors, such as CCL5, CXCL12, which in turn exert paracrine actions on breast cancer cells that resulted in their invasion and/or distant organ metastasis. ${ }^{9}$ Among them, CXCL12 has been widely investigated in breast cancer metastasis. ${ }^{17}$ Beside its role in supporting tumour growth and angiogenesis, CXCL12 was demonstrated to be important in helping select metastatic tumour cells for bone metastasis. More interestingly, breast cancer cells with high expression of CXCR4 and CXCR7, the chemokine receptors for CXCL12, are apt to migrate to the distant sites where CXCL12 is highly expressed. ${ }^{18,19}$ Systemically blocking CXCR4 with specific antagonists can impair metastasis of breast tumour cells to the lung. ${ }^{20}$ However, the role of $\mathrm{CXCL} 12$ produced by MSCs in tumour metastasis remains unclear.

It has been reported that CXCL12 produced by MSCs can be inhibited in presence of TGF $\beta$, indicating that the potential role of TGF $\beta$ in regulating tumour metastasis may be mediated at least in part through MSCs. ${ }^{21}$ Indeed, TGF $\beta$ that is highly produced by MSCs, tumour cells, as well as suppressive immune cells can facilitate tumour cell migration and invasion by the induction of the EMT process of tumour cells. ${ }^{22}$ Classically, TGF $\beta$ binds to the type 2 TGF $\beta$ receptor (TGFBR2), causing the recruitment and phosphorylation of TGFBR1, and then activates the downstream signalling. Our previous study found that TGF $\beta$ promoted T-cell

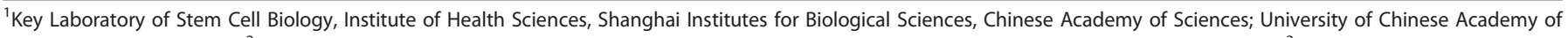

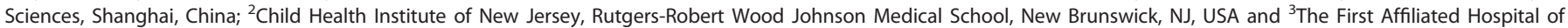

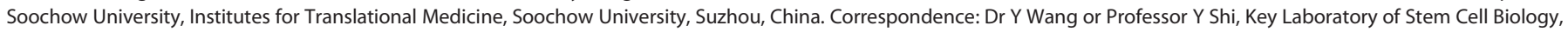

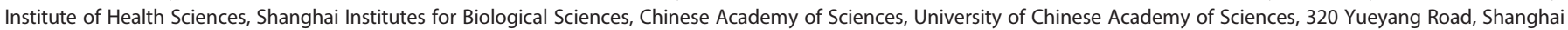
200031, China.

E-mail: yingwang@sibs.ac.cn or yufangshi@sibs.ac.cn

${ }^{4}$ These authors are co-senior authors.

Received 3 February 2016; revised 15 April 2016; accepted 11 May 2016; published online 26 September 2016
} 
immune responses in the presence of MSCs through inhibiting inflammatory cytokine-induced inducible NO synthase (iNOS) expression in MSCs in vitro. ${ }^{23}$ However, in tumour metastasis, the interplay of MSCs and TGF $\beta$ in regulating tumour metastasis has not been well studied.

In the current study, we found that the prometastatic effect of MSCs in breast cancer model was abolished when the TGF $\beta$ signalling pathway was blocked in MSCs, suggesting the pivotal roles of TGF $\beta$-mediated regulation of MSCs on tumour metastasis. We found that TGF $\beta$ downregulated the expression of CXCL12, which restricted tumour metastasis. Double knock-down of the expression of TGFBR2 and CXCL12 in MSCs was shown to reverse the effect of TGF $\beta$-unresponsive MSCs on tumour metastasis to the lung, providing the evidence of the role of TGF $\beta-C X C L 12$ axis in tumour metastasis. More importantly, this regulation of tumour metastasis by CXCL12 was related to its regulation of CXCR7 in 4T1 cells. Blocking of CXCR7 expression in 4T1 cells by shRNA inhibited breast cancer metastasis. Thus, our data demonstrated that $\mathrm{CXCL} 12$ produced by MSCs in the primary tumour site impedes breast cancer metastasis via inhibiting CXCR7 expression in 4T1 cells, however, TGF $\beta$ can diminish the restriction of MSCproduced $\mathrm{CXCL} 12$ on tumour cells. We also described that upregulation of TGF $\beta$ and CXCR7, and repression of CXCL12 are prominent features of aggressive clinical breast cancer, and we found that they are prognostic parameters for patient survival, indicative of their critical roles in breast cancer pathogenesis. Our study implicates a role for a TGF $\beta-C X C L 12-C X C R 7$ regulatory network between MSCs and tumour cells in breast cancer metastasis.

\section{RESULTS}

Promotion of tumour metastasis by MSCs is dependent on TGF $\beta$ signalling

MSCs recruited to tumours comprise a predominant, multifunctional cell type in the tumour microenvironment, modulating differentiation events in tumour cells, regulating immune responses and maintaining different stages of tumour development. These functions are dependent on their potential to produce various immunoregulatory factors, chemokines, growth factors, induced secondary to their stimulation by inflammatory cytokines. ${ }^{15}$ Among these cytokines, TGF $\beta$ exhibits paradoxical effects on tumours, inhibiting tumour growth at the early benign stage, while promoting tumour progression in advanced stage via enhancing the EMT process. ${ }^{24}$ TGF $\beta$ can be produced by tumour cells and suppressive immune cells, as well as MSCs. We previously demonstrated that ablating TGF $\beta$ signalling in MSCs resulted in enhanced T-cell activity, ${ }^{23}$ however, the interactions between TGF $\beta$ and MSCs and their effects on tumour progression remains unclear. As described in our previous study, murine MSCs highly expressed the TGF $\beta$ receptor 2 (TGFBR2), a key membrane-bound receptor subunit for transmitting TGF $\beta$ signalling (Figure 1a). Once activated by TGF $\beta$, phosphorylated SMAD3, a classical downstream molecule of TGF $\beta$ pathway, could be observed in MSCs (Figure $1 \mathrm{~b}$ ). We further generated TGF $\beta$ unresponsive MSCs by transfecting MSCs with the plasmid encoding a dominant negative form of TGFBR2 (dn-TGFBR2) to abolish TGF $\beta$ signalling. Silencing TGFBR2 in MSCs resulted in loss of the phosphorylation of SMAD3 upon TGF $\beta$ treatment (Figure 1b). However, dn-TGFBR2 MSCs and dn-control MSCs showed similar morphology and comparable proliferation ability (Supplementary Figures 1a and b).

To further test the biological function of the interaction between TGF $\beta$ and MSCs in tumour progression, we injected 4T1 cells, a well-characterized murine metastatic breast cancer cell line, together with dn-control MSCs or dn-TGFBR2 MSCs into the fat pad of BALB/c mice. Compared with the control group with 4T1 cell injection alone, neither dn-control MSCs nor dn-TGFBR2
MSCs promoted growth of the primary tumour (Figure 1c; Supplementary Figure 1c). In contrast, dn-control MSCs markedly promoted the formation of lung metastases at day 28. Surprisingly, lung metastases were hardly detected in mice injected with dn-TGFBR2 MSCs and 4T1 cells (Figures 1d and e). The survival analysis showed that dn-TGFBR2 MSCs enhanced the survival of $4 \mathrm{~T} 1$ tumour-bearing mice (Figure 1f). Considering the possibility that TGF $\beta$ promoted T-cell responses in the presence of MSCs, we studied the 4T1 tumour model with MSC co-injection in the non-obese diabetic/severe combined immunodeficiency (NOD/SCID) mice. Similar to the tumour model in immunocompetent mice, the primary tumour size showed no significant difference (Supplementary Figure 2a). The promotion of lung metastasis by dn-control MSCs was abolished by silencing TGFBR2 in MSCs (Figure 1g; Supplementary Figure 2b), indicating that the effects of TGF $\beta$ and MSC interactions on tumour metastasis is independent of their modulation on T cells.

Because increased tumour cell colonization can be related to enhanced tumour metastasis, we further explored the possibility that the interaction between TGF $\beta$ and MSCs affected the localization of tumour cells in secondary organs with subsequent colonization. ${ }^{25}$ To study this, 4T1 cells were co-injected intravenously with dn-control MSCs or dn-TGFBR2 MSCs into mice. Four weeks later, the lungs of these mice were analysed. We found that both dn-control MSCs and dn-TGFBR2 MSCs increased 4T1 cell colonization to the lung at comparable levels (Figure 1h), suggesting that the influence of TGF $\beta$ and MSC interactions on tumour metastasis focused on the early stages of the invasion and metastasis cascade.

TGF $\beta$ suppresses CXCL12 expression in MSCs

Previous reports suggested that cytokines secreted by MSCs, such as VEGF, IL-6, CCL2, CCL5, promoted tumour growth and metastasis. $^{9,15,26}$ We wondered if TGF $\beta$ can modulate the expression of these key factors. Gene expression profiles of MSCs with or without TGF $\beta$ treatment were detected and analysed. Twenty-seven genes that were grouped to cytokine-cytokine receptor interaction pathway were significantly affected by TGF $\beta$ (Supplementary Table 1). Among them, we chose CXCL12 (Supplementary Figure 3a) as a candidate because it was previously shown to correlate with tumour metastasis through providing a signal for tumour cells reaching the distant metastatic organ. ${ }^{19}$ Indeed, CXCL12 was expressed at high levels in MSCs (Figures $2 \mathrm{a}$ and b). Interestingly, our chemokine qPCR-array data showed that the extremely high level of CXCL12 in MSCs was markedly downregulated in the presence of TGF $\beta$ (Figure 2a). TGF $\beta$ also suppressed CXCL12 expression at the protein level (Supplementary Figure 3b). Furthermore, TGF $\beta$ regulated CXCL12 expression in a dose-dependent manner (Supplementary Figure $3 \mathrm{C}$ ). The TGF $\beta$-induced suppression of CXCL12 at both the mRNA and protein levels could be reversed by SB431542, a specific inhibitor of the TGF $\beta$ receptor (Figures $2 c$ and $d$ ). Consistently, TGF $\beta$ treatment had only a marginal effect on CXCL12 levels in dn-TGFBR2 MSCs (Figure 2e). In addition, all three TGF $\beta$ isoforms, TGF $\beta 1, T G F \beta 2$ and TGF 33 , inhibited CXCL12 production (Supplementary Figure 3d).

TGF $\beta$, which is produced by tumour cells, MSCs, as well as immune cells, is a pleiotropic cytokine with plentiful expression in tumours, which regulates tumour progression. ${ }^{22}$ Given the negative effects of TGF $\beta$ on CXCL12 expression in in vitro expanded MSCs, we further questioned if MSCs derived from tumours exhibit low levels of CXCL12. Compared with MSCs derived from bone marrow or lung, tissues that are potential target for metastatic tumour cell localization, 4T1 tumourderived MSCs showed only low expression of CXCL12 mRNA (Figure 3a). Interestingly, high levels of CXCL12 mRNA in bone marrow-derived MSCs could be suppressed in presence of $4 \mathrm{~T} 1$ 

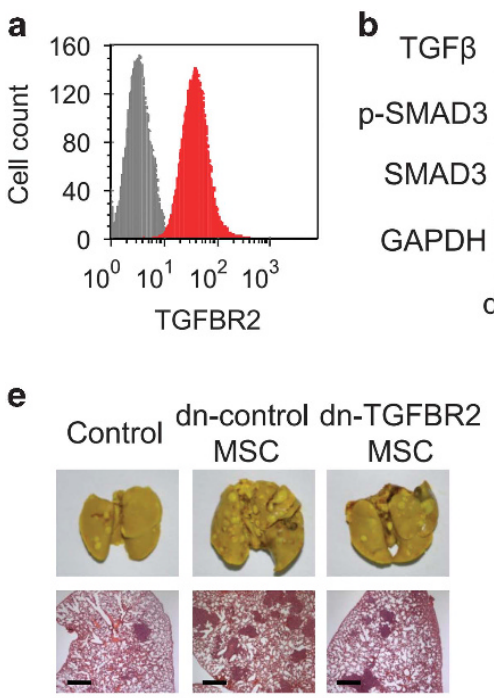

f

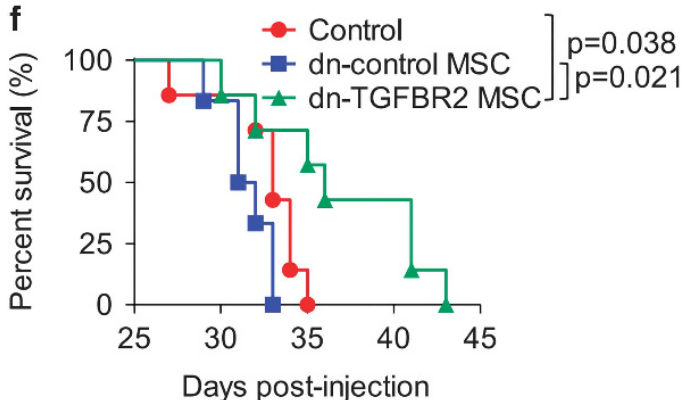

C

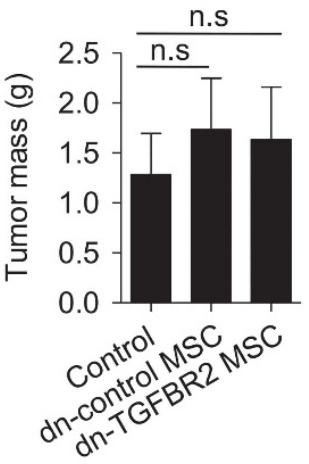

g

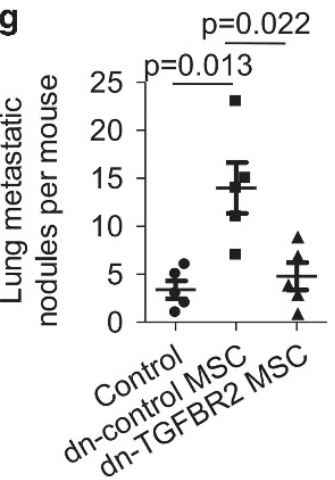

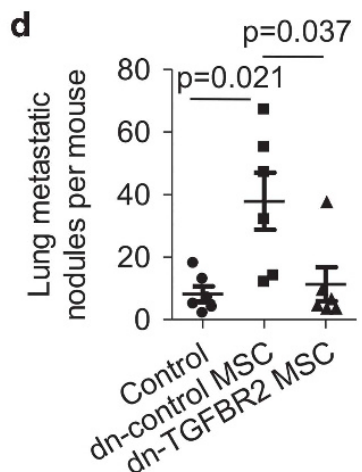

h

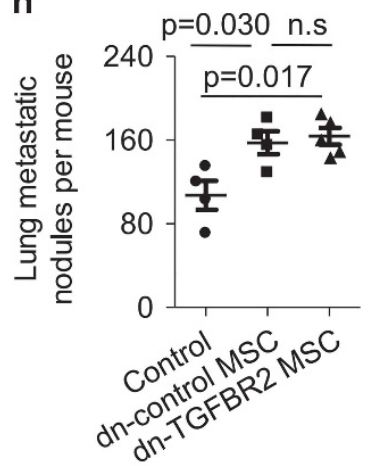

Figure 1. MSCs promote 4T1 breast cancer metastasis in a TGF $\beta$-dependent manner. (a) TGFBR2 expression on MSCs was analysed by flow cytometry. Isotype control and TGFBR2 were labelled grey or red colours, respectively. (b) MSCs were transfected with control vector (dncontrol MSC) or TGFBR2dn plasmid (dn-TGFBR2 MSC). Cells were treated with or without TGF $\beta$ for 30 min and assayed for SMAD3 and p-SMAD3 expression by western blot analysis. GAPDH was used as a loading control. (c-e) 4 T1 breast cancer cells $\left(4 \times 10^{5}\right)$ were co-injected with dn-control or dn-TGFBR2 MSCs $\left(2 \times 10^{5}\right)$ into fat pad of BALB/c mice; control group received 4T1 cells alone ( $n=6$ each). Tumours were excised and weighed after 4 weeks (c); metastatic nodules in the lungs were quantified (d) and shown by whole-lung images (stained by Bouin's solution); and H\&E staining (scale bars, $500 \mu \mathrm{m})(\mathbf{e})$. (f) The survival curve of tumour bearing mice. BALB/c mice received 4T1 cells alone or together with MSCs as described in (c). (g) $4 \mathrm{~T} 1$ breast cancer cells $\left(4 \times 10^{5}\right)$ were co-injected with different kinds of MSCs $\left(2 \times 10^{5}\right)$ into fat pad of NOD/SCID mice; control group received 4T1 cells alone ( $n=5$ each). Four weeks later, metastatic nodules on the lung were quantified. (h) $4 \mathrm{~T} 1$ breast cancer cells $\left(1 \times 10^{5}\right)$ were intravenously injected to BALB/c mice with or without dn-control or dn-TGFBR2 MSCs $\left(5 \times 10^{4}\right)$. Three weeks later, metastatic nodules on the lung were quantified. Data were shown as means \pm s.e.m. NS, no significance.

tumour-cultured supernatants. Suppression of CXCL12 by tumourcultured supernatants was reversed in presence of SB431542, indicating that TGF $\beta$ in tumour microenvironment is critical in regulating CXCL12 expression in MSCs (Supplementary Figure 4).

CXCL12 produced by MSCs suppresses breast cancer metastasis To verify the role of the negative regulation of CXCL12 expression by TGF $\beta$ in tumour metastasis, we generated MSC cell lines with CXCL12 knockdown by lentivirus transfection in both dn-control MSCs and dn-TGFBR2 MSCs. These cells exhibited a significant reduction of CXCL12 mRNA and protein (Supplementary Figure 5a; Figure $3 b$ ). We inoculated 4T1 cells with different types of MSCs into the mouse fat pad. No difference in primary tumour size was observed (Supplementary Figure 5b). As described above, blocking TGF $\beta$ receptor function in MSCs suppressed the metastatic nodules in the lungs. Interestingly, in dn-TGFBR2 MSCs-injection groups, CXCL12 knockdown promoted tumour metastasis, showing a similar number of metastatic nodules in the lungs as dn-control MSCs (Figures $3 c$ and $d$ ). This result suggests that dn-TGFBR2 MSCs regulate tumour metastasis in a CXCL12dependent manner.

Many studies reported the critical role of CXCL12 in mediating breast cancer cell metastasis, including functions in preselecting the seed for bone metastasis or providing the chemokine gradient for tumour cell migrate to the distant organ with high levels of CXCL12. ${ }^{27,28}$ The major function of CXCL12 in breast cancer metastasis to the lung was demonstrated by employing specific antagonists to block CXCR4, which is one of the key receptors for CXCL12. ${ }^{20}$ However, no direct evidence has demonstrated the role of CXCL12 produced by MSCs in tumour metastasis. To achieve this, we generated a MSC cell line stably overexpressing CXCL12. We then inoculated 4T1 cells, together with MSCs continuously expressing CXCL12 into BALB/C mouse. There was no difference in primary tumour size (Supplementary Figure 5c). However, decreased tumour nodules in the lungs were observed in CXCL12-overexpressing MSC group compared with those of control MSCs group, implicating that CXCL12 expression restricts breast cancer cell metastasis to the lungs (Figures $3 e$ and $f$ ). Together these data suggest that CXCL12 derived from MSCs acts as a negative regulator during breast cancer metastasis.

CXCL12 downregulates CXCR7 expression on tumour cells

To further investigate the role of $\mathrm{CXCL} 12$ in negatively modulating the metastatic activity, we determined the expression of CXCL12 and its receptors, such as CXCR4 and CXCR7 in tumour cells. We found that the $4 \mathrm{~T} 1$ cell line did not express CXCL12 and only 
a

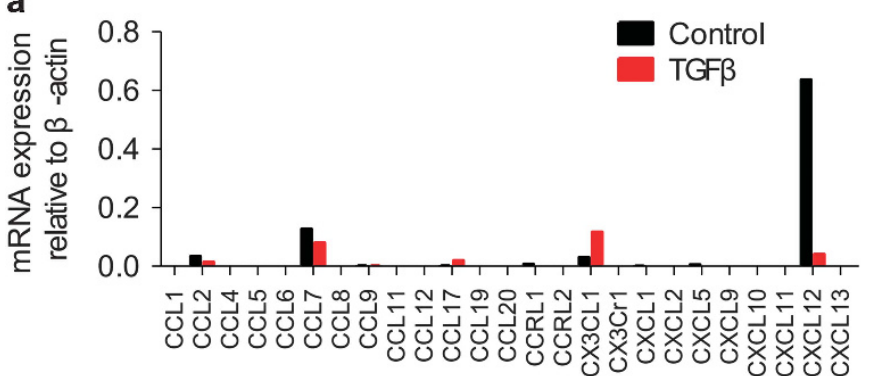

C

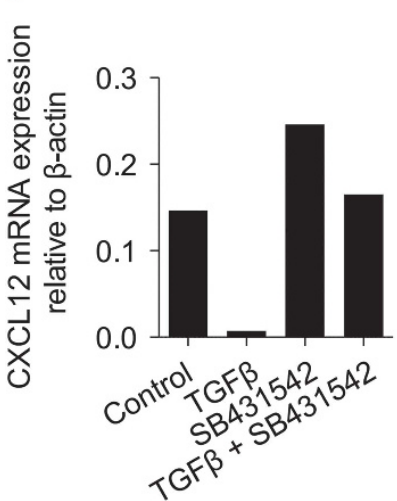

d

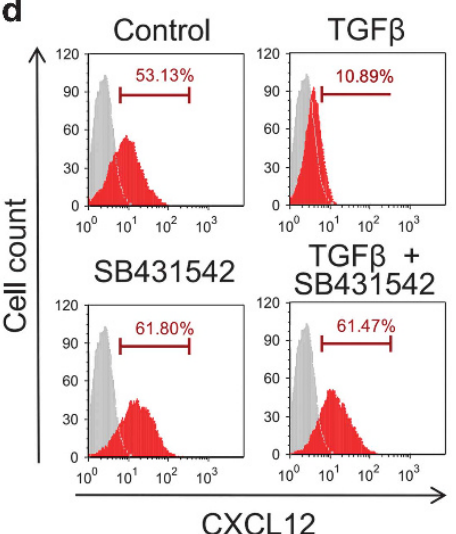

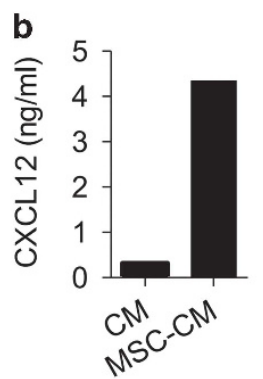
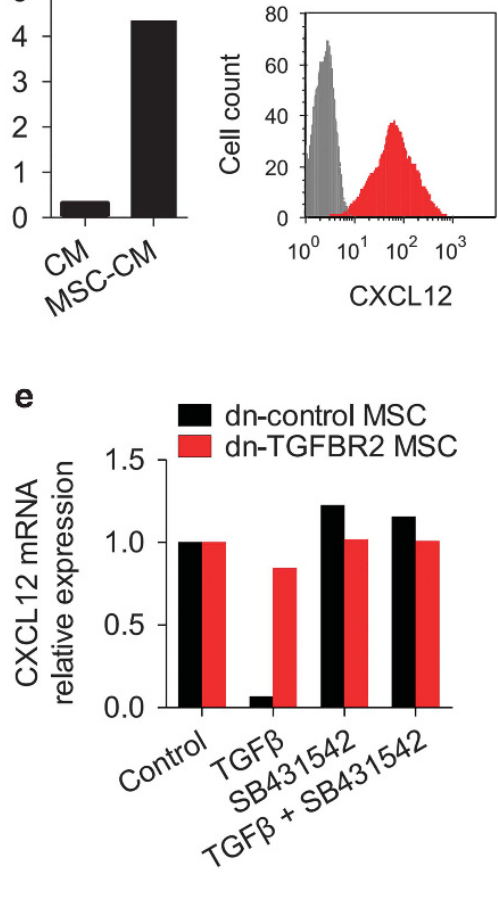

Figure 2. TGF $\beta$ downregulates CXCL12 expression in MSCs. (a) Chemokine expression profile of MSCs in the presence of TGF $\beta$. MSCs were stimulated with TGF $\beta(10 \mathrm{ng} / \mathrm{ml})$ for $12 \mathrm{~h}$ and assayed for mRNA expression of chemokines. (b) CXCL12 expression in MSCs was determined by ELISA and flow cytometry. CM, conditioned medium. (c, d) TGF $\beta$ suppresses CXCL12 expression in MSCs. MSCs were stimulated with TGF $\beta$ $(10 \mathrm{ng} / \mathrm{ml})$ for $12 \mathrm{~h}$. SB431542 $(10 \mu \mathrm{M})$, a TGF $\beta$ receptor antagonist, was used to block TGF $\beta$ signal. CXCL12 expression at both mRNA and protein levels was assayed by real-time PCR (c) and flow cytometry (d), respectively. (e) Blocking TGF $\beta$ receptor reverses the TGF $\beta$-induced suppression of CXCL12 in MSCs. Dn-control or dn-TGFBR2 MSCs were stimulated with TGF $\beta$ (10 ng/ml) for $12 \mathrm{~h}$, in presence of SB431542 $(10 \mu \mathrm{M})$. CXCL12 mRNA expression was determined with real-time PCR.

marginally expressed CXCR4, the classical receptor of CXCL12 (Figures $4 a$ and b). However, the 4T1 cell line expressed high levels of CXCR7 mRNA and protein (Figures $4 a$ and b). CXCR7, also known as RDC1, is a novel defined chemokine receptor for both CXCL12 and CXCL11. ${ }^{29}$ A series of studies have demonstrated that CXCR7 is important in tumour development via regulating tumour cell survival and adhesion. ${ }^{29-31}$ Pretreatment of $4 \mathrm{~T} 1$ cells with MSC-conditioned medium gradually impaired CXCR7 expression during culture process (Figure $4 \mathrm{C}$ ). To determine whether the downregulation of CXCR7 in 4T1 cells depends on MSC-derived CXCL12, CXCL12 neutralizing antibody was added to the culture system of $4 \mathrm{~T} 1$ and MSC-conditioned medium. We found that blocking CXCL12 function can reverse the suppressive effect on CXCR7 expression in 4T1 cells by MSC-cultured medium, suggesting that tumour cell metastasis in this system was predominantly regulated by the CXCL12/CXCR7 axis (Figure 4d).

We further examined whether CXCR7 expression on tumour cells has a dominant role in determining the tumour metastasis. Using lentivirus transfection system to deliver CXCR7 shRNA, we successfully silenced CXCR7 expression in 4T1 cells, seen as significantly suppressed CXCR7 expression at both the mRNA and protein levels, compared with that of 4T1 cells transfected with control shRNA (Supplementary Figures $6 a$ and b). We implanted $4 \mathrm{~T} 1$-control or $4 \mathrm{~T} 1$-sh-CXCR7 cells into the mammary fat pad of female BALB/c mice. Consistent with their comparable in vitro proliferation rates (data not shown), the size of the primary tumour formed by these two cell populations was similar (Figure 4e). However, tumour metastatic nodules in the lungs were much lower in the 4T1-sh-CXCR7 group than in the 4T1control group, suggesting that CXCR7 is critical in regulating tumour metastasis to the lungs (Figure 4f). Taken together, MSCs regulate breast cancer metastasis through forming a paracrine loop with tumour cells, resulting in modulating carcinoma cells to metastasize.

Regulation of the TGF $\beta-C X C L 12-C X C R 7$ axis in MSCs and tumour cells can be observed in humans

To correlate our data from animal experiments with human malignancy, we examined CXCL12 expression in human MSCs. We compared different tissue-derived MSCs and found that adiposederived MSCs (ADSCs) and amniotic fluid-derived MSCs (AMSCs) had a relatively high level of CXCL12 expression compared with umbilical cord-derived MSCs (UCSCs) (Figure 5a). Similarly, treatment MSCs with TGF $\beta$ suppressed the expression of CXCL12 mRNA on both ADSCs and AMSCs, while the presence of SB431542, the TGF $\beta$ inhibitor, could reverse this effect (Figures $5 \mathrm{~b}$ and $\mathrm{c}$ ). These data demonstrate that the negative regulation of CXCL12 expression by TGF $\beta$ can be observed in human MSCs.

We further verified whether the regulation of CXCR7 by CXCL12 could be observed in human breast cancer cells. We analysed the expression of CXCL12 in a panel of human mammary carcinoma cell lines, including highly aggressive cells such as MDA-MB-231 and MDA-MB-435, less aggressive cells such as MCF10AT, MCF7 and T-47D. We found that most of the cell lines did not or only minimally expressed CXCL12 (Supplementary Figure 7a; Figure $5 d$ ). In these cell lines, MCF7 cells showed high expression of CXCR7 but only minimal expression of CXCR4 (Supplementary Figure $7 b$; Figure $5 d$ ). Consistent with results in mouse $4 T 1$ cells, ADSC-specific medium could inhibit the expression of CXCR7 in 


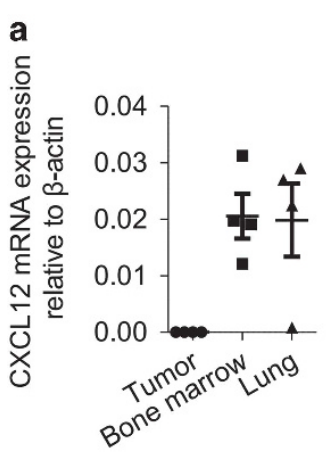

b

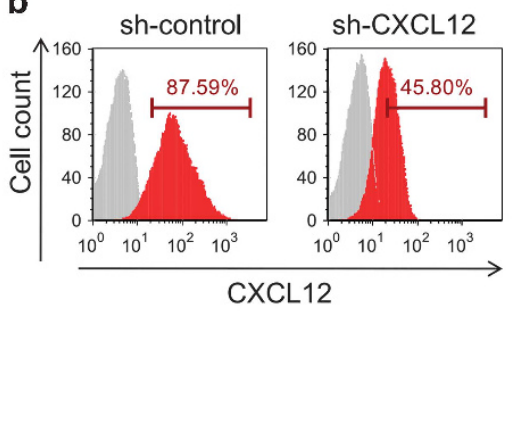

d

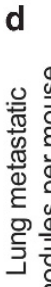

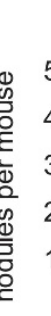

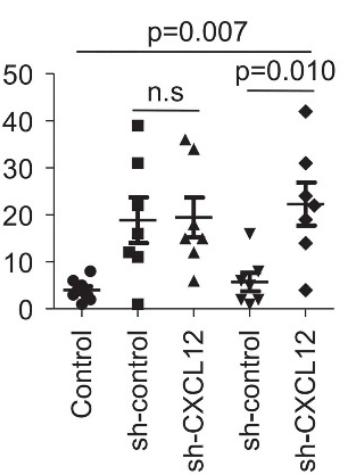

dn-control dn-TGFBR2

MSC MSC e

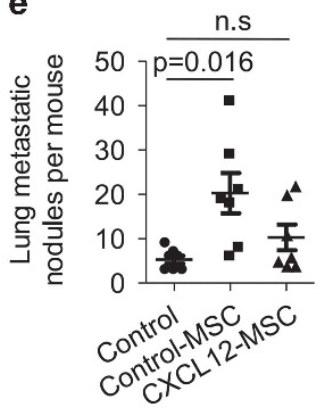

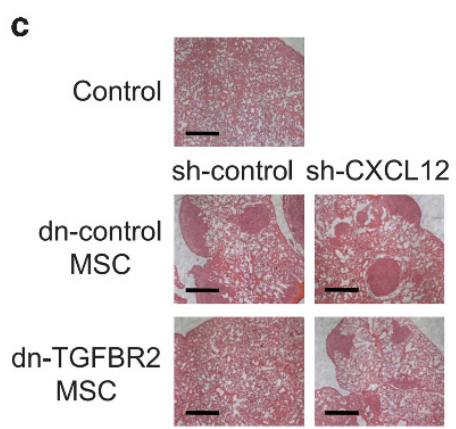

f

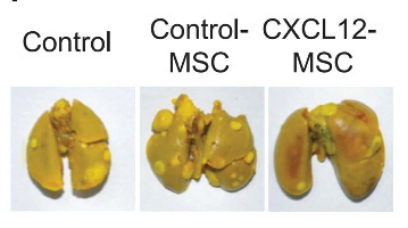

Figure 3. CXCL12 produced by MSCs restricts breast cancer metastasis. (a) Tumour, bone marrow, or lung derived-MSCs were isolated from 4T1 tumour-bearing BALB/c mice $(n=4)$ and were cultured in vitro for $5 \mathrm{~d}$. Cells were assayed for CXCL12 expression by real-time PCR. (b) MSCs were transduced with lentivirus carrying CXCL12 shRNA. Knock down efficiency was determined by flow cytometry. (c, d) Effect of CXCL12 knockdown in TGF $\beta$-unresponsive MSCs on breast cancer metastasis on the lungs. Dn-control MSCs or dn-TGFBR2 MSCs were transduced with shRNA CXCL12-containing lentivirus (sh-CXCL12). MSCs $\left(2 \times 10^{5}\right)$ were co-injected with 4 T1 breast cancer cells $\left(4 \times 10^{5}\right)$ into fat pad of BALB/C mice; control group received 4T1 cells alone ( $n=7$ each). Four weeks later, metastatic nodules on the lung were examined by H\&E staining (scale bars, $700 \mu \mathrm{m})(\mathbf{c})$ and quantification (d). (e, f) Overexpressing of CXCL12 in MSCs restricts breast cancer metastasis to the lungs. MSCs were transduced with lentivirus carrying the gene encoding CXCL12. 4T1 breast cancer cells $\left(4 \times 10^{5}\right)$ were co-injected with control or CXCL12overexpression MSCs $\left(2 \times 10^{5}\right)$ into fat pad of BALB/c mice ( $n=7$ each). Four weeks later, metastatic nodules in the lung were counted (e). Metastatic nodules in the lung were shown by whole-lung images (stained by Bouin's solution) (f). Data are shown as means \pm s.e.m. NS, no significance.

MCF7 cells. In addition, recombinant human CXCL12 could suppress CXCR7 expression on MCF7 cells (Figure 5e). Therefore, CXCL12 regulates CXCR7 expression in human breast cancer cells.

CXCL12 and CXCR7 expressions are correlated with poor prognosis of breast cancer in clinical samples

To extend these findings to a clinical context, we tested the hypothesis that the level of TGF $\beta$, CXCL12 and CXCR7 expression in breast tumours could contribute to the clinical outcome. Expression of these three genes was measured in 33 breast cancer patients using quantitative reverse transcription PCR (qRT-PCR). mRNA expression of TGF $\beta$ in tumours was higher than that of paired normal tissues, while CXCL12 mRNA expression in tumours was markedly lower level than the paired normals (Figure 6a). Segregating breast tumours into two groups based on high or low expression of CXCL12 (median levels), we found that the expression of both TGF $\beta$ and CXCR7 was significantly decreased in the CXCL12 high group as compared with the CXCL12 low group, indicating that there is a negative correlation between TGF $\beta$, CXCR7 and CXCL12 expression in breast cancer (Figure 6b).

We also used a second approach to analyse potential clinical implications of the CXCL12-CXCR7 axis that we have identified. We used an online database of potential breast cancer biomarkers (http://www.kmplot.com) to assess the prognostic implications of CXCL12 and CXCR7 expression in breast cancer patients. Patients whose tumours expressed lower levels of CXCL12 exhibited poor relapse-free survival and distant metastasis-free survival (Figures $6 c$ and d). Expression of high levels of CXCR7 was also correlated with poor relapse-free, and distant metastasis-free survival (Figures $6 \mathrm{e}$ and f). These results indicate that changes of CXCL12 and CXCR7 are significant indicators for the aggressive breast cancer.

\section{DISCUSSION}

Many studies have demonstrated that MSCs are critical in supporting and maintaining the metastatic capability of tumour cells. These studies have utilized multiple different approaches, such as reconstitution of tumour extracellular matrix structures and exchanging the signals with tumour cells. Yet, the potential mechanism needs to be further studied. Our study showed that TGF $\beta$ signalling has a dominant role in MSC-mediated promotion on breast cancer lung metastasis. The action of TGF $\beta$ on MSCs decreased CXCL12 production, which modulated the sequential CXCR7 expression on breast cancer cells and their metastasis (Figure 7).

A paracrine loop was formed between MSCs and tumour cells, resulting in tumour cell invasion and metastasis. MSCs resident in the tumour lead to the recruitment of inflammatory cells, such as $T$ cells, monocytes, that contribute to the intravasation process of tumour metastasis. ${ }^{15}$ We found that blocking TGF $\beta$ signalling in MSCs can inhibit $4 \mathrm{~T} 1$ cell metastasis to the lungs. Although our 
a

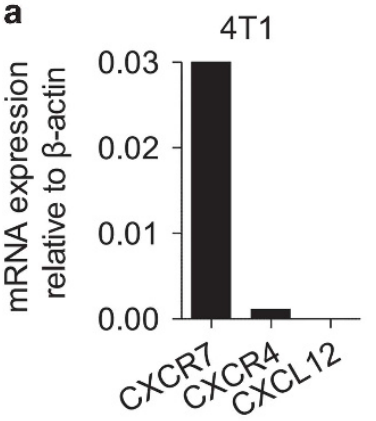

d

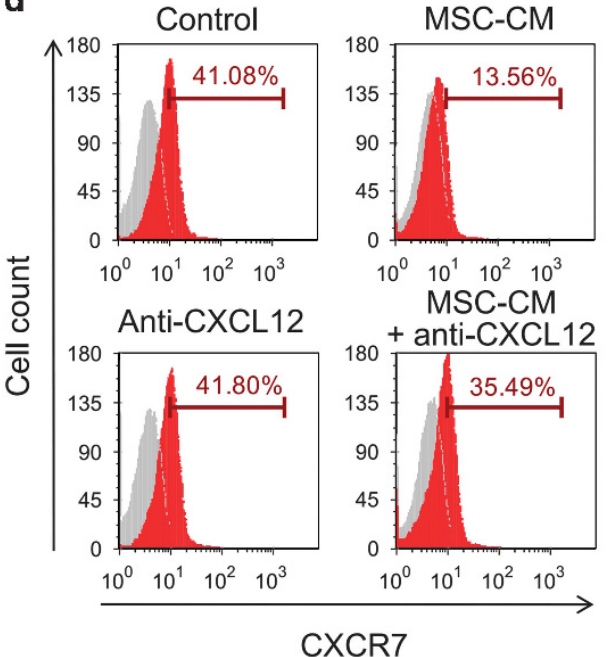

b

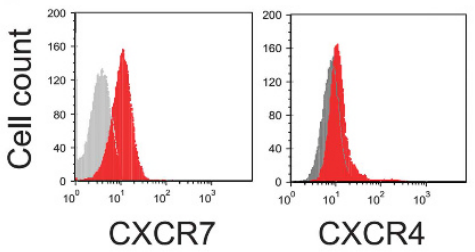

C

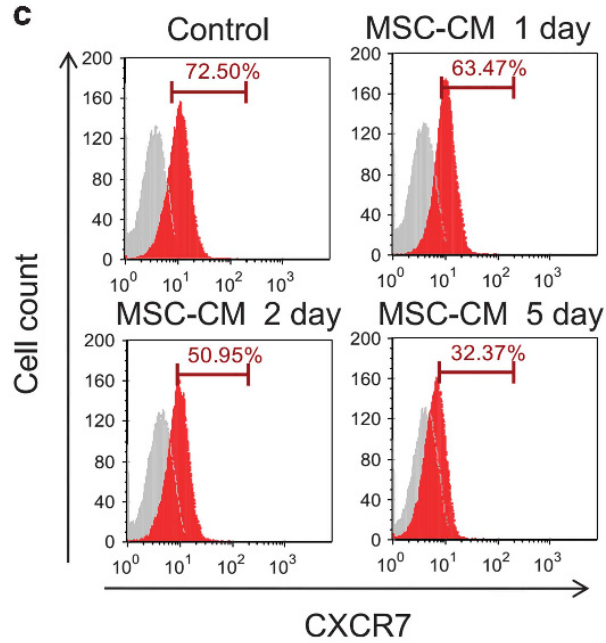

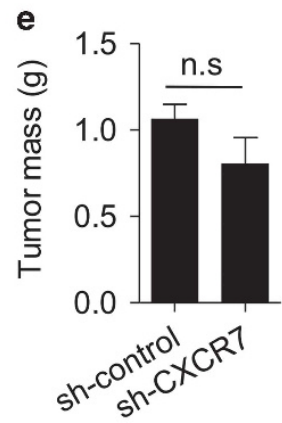

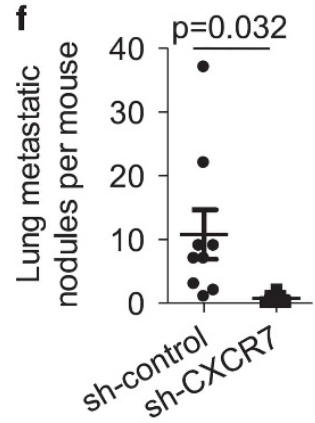

Figure 4. CXCL12 downregulates CXCR7 expression on tumour cells. (a, b) The expression of CXCR4 and CXCR7 in cultured 4T1 tumour cells was analysed by real-time PCR (a) and flow cytometry (b), respectively. (c) 4T1 cells were treated with MSC-conditioned medium for the indicated times. CXCR7 expression on 4T1 cells was analysed by flow cytometry. (d) 4T1 cells were treated with MSC-conditioned medium for $3 \mathrm{~d}$ in presence of CXCL12 neutralizing antibody. CXCR7 expression on tumour cells was analysed by flow cytometry. (e, f) Knockdown of CXCR7 in 4T1 cells results in suppressed breast cancer metastasis on the lungs. 4T1 breast cancer cells were transfected with shRNA targeting CXCR7 or with a non-targeting shRNA control, then cells $\left(8 \times 10^{5}\right)$ were injected into fat pad of BALB/C mice. Primary tumours were excised after 4 weeks and weighed (e). Metastatic nodules in the lung were counted (f). Data were shown as means \pm s.e.m. NS, no significance.

previous study reported that MSCs with deficiency in TGF $\beta$ signalling resulted in the enhanced T-cell responses under IFNY and TNFa stimulation, ${ }^{23}$ immunoregulation was dispensable in the suppression of tumour metastasis by TGF $\beta$-unresponsive MSCs. The promotion of tumour metastasis by the action of TGF $\beta$ on MSCs was associated with the reduction of CXCL12, as TGF $\beta$ unresponsive MSCs with CXCL12 suppression regained the ability to promote $4 \mathrm{~T} 1$ cell metastasis to the lungs. This was consistent with previous results that TGF $\beta$ regulated $\mathrm{CXCL} 12$ expression on MSCs and subsequently influenced bone marrow cell migration and adhesion in vitro. ${ }^{21}$ We need further studies to discover the underlying mechanism. It has been reported that TGF $\beta$ mediated its inhibitory effect on CXCL12 by upregulation of miR-23a. ${ }^{32}$ In addition, the regulation of CXCL12 by TGF $\beta$ could involve in an epigenetic mechanism, as previous study has shown that breast cancer cells were hypermethylated in their CXCL12 promoter region. ${ }^{33}$ We also found that promoters of multiple chemokines were hypermethylated in tumour-derived MSCs, compared with that of bone marrow MSCs (data not shown).

Previous studies have shown that $\mathrm{CXCL} 12$ promoted tumour cell survival, proliferation, angiogenesis, migration through the CXCL12-CXCR4 signalling pathway, and even recruitment of bone marrow cells to the tumour microenvironment. ${ }^{17,34,35}$ Despite others have shown that blocking CXCL12-CXCR4 axis with CXCR4 antagonist could inhibit breast cancer bone metastasis, our study discovered that CXCL12 produced by local MSCs did restrict the metastasis of breast cancer cells to the lung. We found that CXCL12 was the key factor related to the role of TGF $\beta$ regulating MSCs on breast cancer metastasis to the lungs. In this process, the level of CXCL12 in MSCs showed a negative correlation with breast cancer cell metastasis. That is, high level of CXCL12 impeded tumour cell metastasis to the lung, whereas low level of CXCL12 regulated by TGF $\beta$ facilitated the metastasis. Indeed, MSCs, especially bone marrow-derived cells, expressed high level of CXCL12. However, tumour-derived MSCs marginally expressed. This can be due to the abundance of TGF $\beta$ in tumour microenvironment, which has the potential to downregulate CXCL12 expression in tumour-derived MSCs and promote tumour metastasis. Consistent with the result that induced CXCL12 expression in breast cancer cells lead to suppression on tumour metastasis, CXCL12-overexpressed MSCs cannot induce the significant metastatic modes in the lungs, indicating that CXCL12 functioned as an inhibitor of tumour metastasis. Detail analysis on the breast cancer patients showed that CXCL12 was closely associated with a better prognosis of breast cancer. These results suggest that the change of CXCL12 in tumour microenvironment could decide the event of tumour metastasis. 

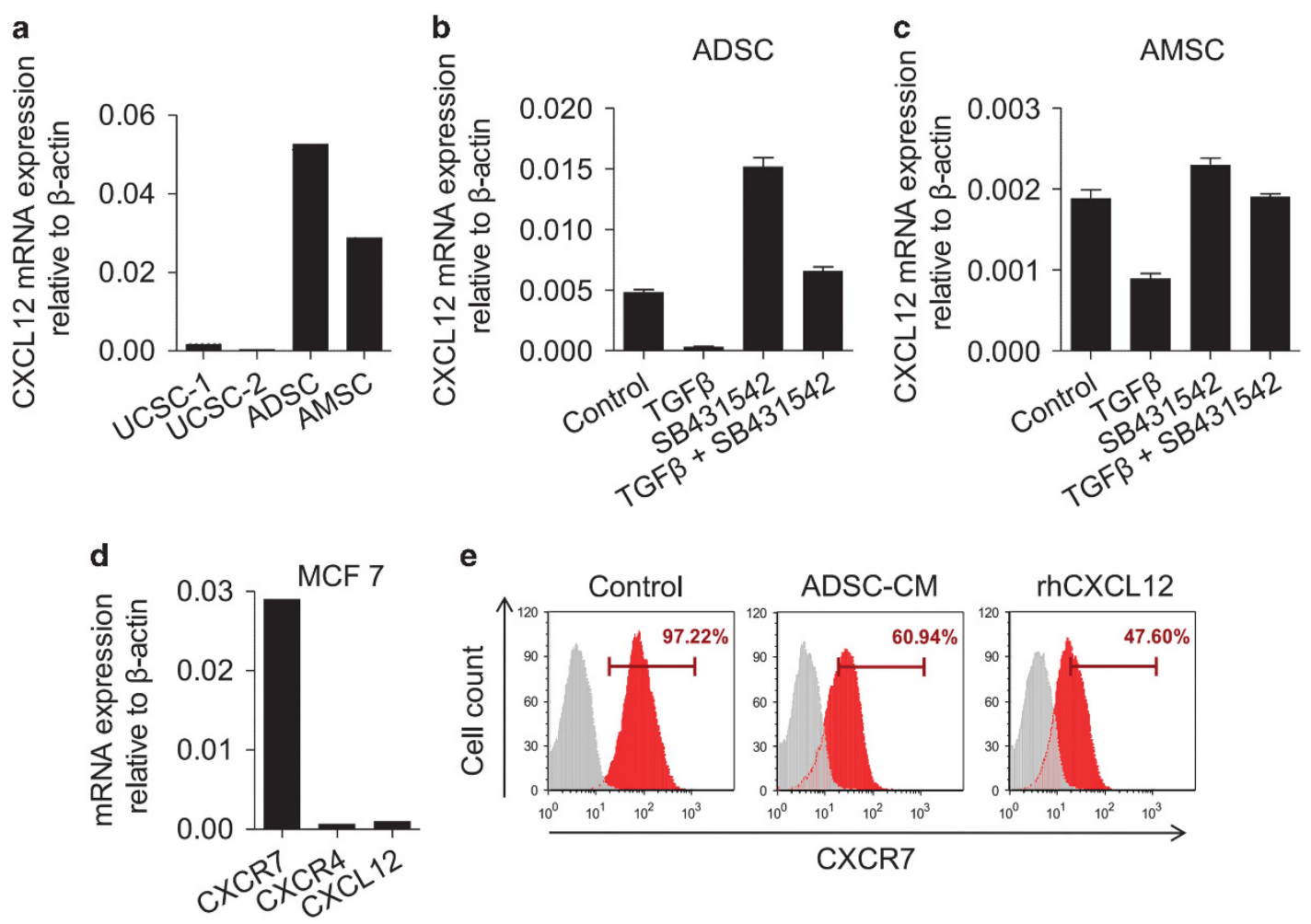

Figure 5. Regulation of the TGF $\beta-C X C L 12-C X C R 7$ axis in MSCs and tumour cells can be observed in human cells. (a) CXCL12 expression in different tissue-derived human MSCs was analysed by real-time PCR. ADSC, adipose-derived MSC; AMSC, amniotic fluid-derived MSC; BMSC, bone marrow-derived MSC; UCSC, umbilical cord-derived MSC. (b, c) TGF $\beta$ inhibits CXCL12 expression in human MSCs. Cultured human ADSCs (b) and AMSCs (c) were stimulated with TGF $\beta(10 \mathrm{ng} / \mathrm{ml})$ for $12 \mathrm{~h}$. SB431542 $(10 \mu \mathrm{M})$ was added to block the TGF $\beta$ receptor. CXCL12 expression was assayed by real-time PCR. Data were shown as means \pm s.e.m. (d) The expression of CXCR4, CXCR7 and CXCL12 in cultured MCF7 cells was analysed by real-time PCR. (e) MCF7 cells were treated with ADSC-conditioned medium or recombinant human CXCL12 protein $(20 \mathrm{ng} / \mathrm{ml})$ for 2 days. Cells were analysed for CXCR7 expression by flow cytometry.

It is generally believed that CXCL12 mediates multiple biological processes via a single cell surface receptor known as chemokine receptor 4 (CXCR4). However, recently an orphan receptor CXCR7 (RDC1) was found to bind with high affinity to CXCL12 and CXCL11. Membrane-associated CXCR7 is expressed on multiple types of tumours, such as breast cancer, colon cancer and hepatocellular carcinoma. ${ }^{29-31}$ Activation by its ligand provides tumour cells with a growth and survival advantage, and increases their adhesion properties. It has been reported that CXCR7 inhibition with small molecule antagonist or RNA interference hinders tumour growth and metastasis in animal models of breast cancer. ${ }^{30}$ Our work found that CXCL12 secreted by MSCs reduced the expression of CXCR7 on the surface of tumour cells. Knockdown of CXCR7 on tumour cells indeed impeded tumour metastasis. In addition, we found that long-term CXCL12 treatment did not decrease mRNA levels of CXCR7 significantly, indicating that the downregulation of CXCR7 expression by CXCL12 could not happen in gene transcription level (data not shown). A previous study on T cells demonstrated that CXCL12 can induce the internalization of CXCR7 in a dose-dependent manner. ${ }^{36}$ In future studies, we will test the possibility that CXCL12 treatment leads to the internalization of CXCR7 on tumour cells with the resultant inhibition of tumour metastasis. Apart from tumour cells, the regulatory effect of CXCL12 on CXCR7 in other types of cells needs further investigation. Recently, CXCR7 deletion in endothelial cells was reported to promote breast cancer metastasis, ${ }^{37}$ which suggests that MSCs may regulate tumour development through multiple ways.

In conclusion, MSCs are central regulators of tumour metastasis. In response to TGF $\beta$ in the tumour microenvironment, they produce less CXCL12, leading to the high levels of CXCR7 in tumour cells and increased tumour metastasis to the lung. This study has provided a distinct mechanism to explain the clinical correlation between reduced expression of CXCL12, increased expression of TGF $\beta$ and CXCR7, and poor metastasisfree survival of breast cancer patients.

\section{MATERIALS AND METHODS}

Mice

BALB/C and NOD-SCID mice were purchased from the Shanghai Laboratory Animal Center of Chinese Academy of Sciences, Shanghai, China, and maintained under specific pathogen-free conditions. Mice were maintained in the vivarium of Shanghai Jiao Tong University School of Medicine. Animals were matched for age and gender in each experiment. The animal protocols for the experiments described in this paper were approved by the Institutional Animal Care and Use Committee of the Institute of Health Sciences, Shanghai Institutes for Biological Sciences of Chinese Academy of Sciences.

Cells

Mouse MSCs were generated from tibia and femur bone marrow or by isolation from lung and tumour digested with type II collagenase (SigmaAldrich, St Louis, MO, USA) using 6-10-week-old mice. Cells were cultured in DMEM supplemented with $10 \%$ FBS, $2 \mathrm{mM}$ glutamine, $100 \mathrm{U} / \mathrm{ml}$ penicillin and $100 \mu \mathrm{g} / \mathrm{ml}$ streptomycin (all from Invitrogen, Carlsbad, CA, USA). Cells were used before 20th passage. Human-derived MSCs, which were isolated from adipose tissue, umbilical cord and amniotic fluid, were used from 6th to 15th passages. Identification of MSCs (both mouse and human) was detected by their capability to differentiate into adipocytes, 


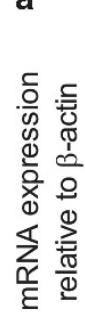

CXCL12

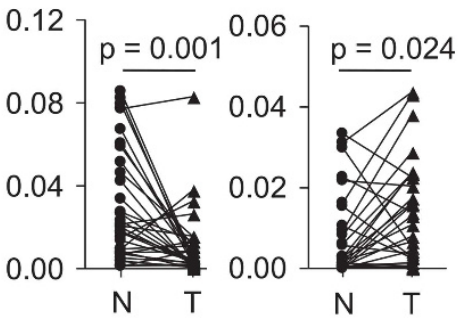

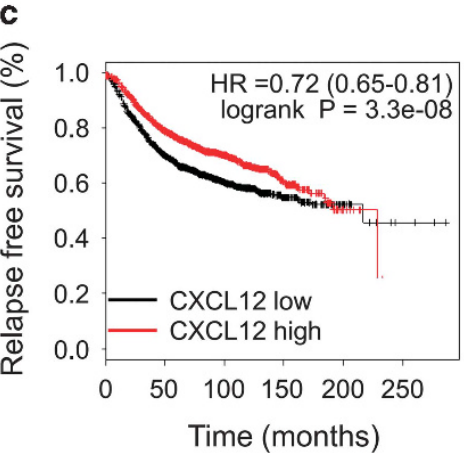

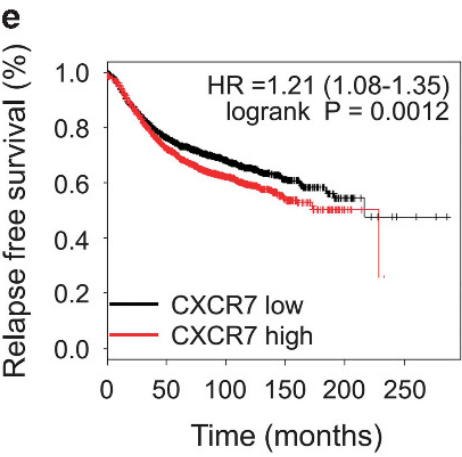

d

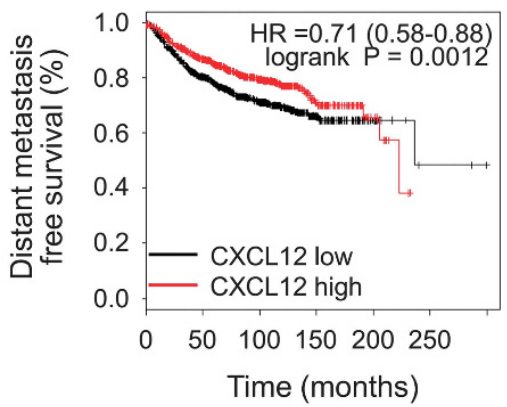

f

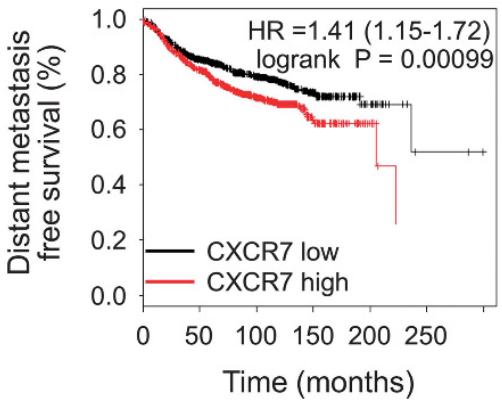

Figure 6. Decreased CXCL12 and elevated CXCR7 are correlated with poor prognosis in clinical breast cancer. (a) The mRNA levels of CXCL12 and TGF $\beta$ in tumour samples were analysed in 33 breast cancer patients. N, normal tissue; T, tumour tissue. (b) The mRNA expression levels of TGF $\beta$ and CXCR7 were compared between CXCL12 low $(L, n=17)$ and CXCL12 high $(H, n=16)$ groups, which were divided according to the median of CXCL12 in the patients. (c, d) Relapse free survival (c) and distant metastasis free survival (d) of breast cancer patients with high- or low-CXCL12 (Affymetrix ID: 209687_at) expression. Hazard ratios (HR) and P-values (log rank P) are depicted for each survival analysis. KaplanMeier survival data were generated using the publicly online tool KM-plotter. (e, f) Relapse free survival (e) and distant metastasis-free survival (f) of breast cancer patients with high- or low-CXCR7 (Affymetrix ID: 212977_at) expression. Hazard ratios (HR) and $P$-values (log rank P) are depicted for each survival analysis. Kaplan-Meier survival data were generated using the publicly online tool KM-plotter.

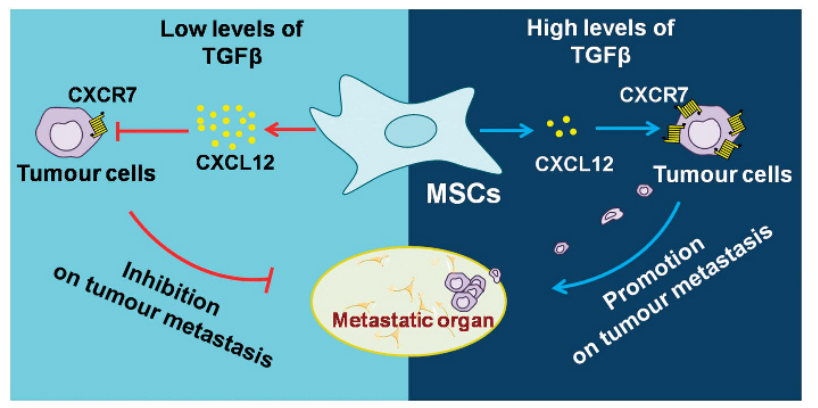

Figure 7. Mechanisms for enhanced tumour metastasis through TGF $\beta$-CXCL12-CXCR7 axis. CXCR7 expressed on breast cancer cells is important for tumour metastasis. MSCs-derived CXCL12 results in decreased expression of CXCR7 in tumour cells, and inhibition of tumour metastasis. However, abundant TGF $\beta$ in the tumour microenvironment efficiently inhibits CXCL12 expression in MSCs. Therefore, TGF $\beta$ blocks CXCR7 reduction by MSCs and promotes subsequent metastasis.

osteoblasts and chondrocytes, and by their expression of specific cell surface markers.

Plasmids and lentivirus particles

The sequence of dn-TGFBR2 was amplified from the genomic DNA of TGF $\beta$ receptor 2-dn transgenic mice and inserted into pLVX-IRES-zsGreen vector. CXCL12 sequence was cloned and inserted into pLVX-IRES-zsGreen vector. CXCL12-specific (sh-CXCL12), CXCR7-specific (sh-CXCR7) and control (sh-control) short hairpin RNA (shRNA) lentivirus particles were prepared by GenePharma (Shanghai, China, http://www.genepharma.com/).
Reagents

Recombinant human TGF $\beta$, CXCL12, anti-mouse TGFBR2-PE, anti-mouse CXCL12-APC, anti-mouse/human CXCR7-APC were purchased from R\&D Systems (Minneapolis, MN, USA). Mouse p-SMAD3, SMAD3 and GAPDH monoclonal antibodies were obtained from Cell Signaling Technology (Danvers, MA, USA). Mouse CXCL12 polyclonal antibody, anti-mouse CXCR4-APC, mouse CXCL12 ELISA-kit and TGF $\beta$ ELISA-kit were from eBiosciences (La Jolla, CA, USA). Bouin's solution and SB431542 were from Sigma-Aldrich.

\section{Animal studies}

MSCs $\left(2 \times 10^{5}\right)$ were co-injected with $4 \mathrm{~T} 1$ tumour cells $\left(4 \times 10^{5}\right)$ into $\mathrm{BALB} / \mathrm{c}$ mice in the intramammary gland fat pad. At various time points, primary tumour sizes were measured and the immune cell frequency in tumours or peripheral blood was analysed by flow cytometry. At day 28 after tumour cell inoculation, the resultant tumours were excised and weighed. Tumour nodules on the lung were counted and showed by H\&E staining.

Real-time PCR

Total RNA was isolated using RNAprep pure Cell/Bacteria Kit (Tiangen Biotech, Beijing, China), and reverse-transcribed into CDNA was performed using 1st cDNA Synthesization Kit with oligo(dT) ${ }_{15}$ (Tiangen Biotech, Beijing, China). Gene probes were used with SYBR Green Master Mix (Roche Diagnostics, Indianapolis, IN, USA) and a 7900HT Fast Real-Time PCR system (Applied Biosystems, Foster City, CA, USA). Total amount of mRNA was normalized to endogenous $\beta$-actin mRNA. Sequences of primer pairs were listed in Supplementary Table 2.

Flow cytometry analysis

Cells surface markers and intracellular cytokines were stained according to the R\&D flow cytometry protocol. Briefly, cells were first collected and 
washed with PBS. For surface staining of CXCR4, CXCR7, cells were resuspended in PBS containing $0.5 \%$ BSA and were incubated on ice for $30 \mathrm{~min}$ with fluorochrome-conjugated antibodies. For intracellular staining of CXCL12, after permeabilization, cells were stained with fluorochromeconjugated antibodies and analyzed on a FACS Calibur (Becton Dickinson, SanJose, CA, USA, http://www.bd.com) according to the manufacturer's instruction. FCS Express software was used for data analysis.

\section{Western blots}

To analyse protein expression, cells were washed with PBS. The cell pellet was resuspended in lysis buffer. After extracting total protein, immunoblotting was performed as follows. Briefly, protein in SDS sample buffer was heated at $95^{\circ} \mathrm{C}$ for $10 \mathrm{~min}$, separated on a gradient SDSpolyacrylamide gels and transferred onto a PVDF membrane. After blocking with $5 \%$ nonfat milk in TBS with $0.1 \%(\mathrm{w} / \mathrm{v})$ Tween-20 for $1 \mathrm{~h}$ at room temperature, western blotting was performed with the following primary antibodies: anti-p-SMAD3, anti-SMAD3, anti-CXCL12 or antiGAPDH. After incubation overnight with primary antibodies at $4{ }^{\circ} \mathrm{C}$, membranes were incubated with a HRP-conjugated secondary antibody. The blot was subjected to chemiluminescent detection according to the manufacturer's instructions.

\section{Clinical samples}

The tumour specimens from breast cancer patients in this study were obtained from the Tumour Center at the Shanghai Institute of Health Sciences. All individuals provided informed consent. The study was approved by the Institutional Biomedical Research Ethics Committee of the Shanghai Institutes for Biological Sciences (Chinese Academy of Sciences).

\section{Statistical analysis}

For statistical analysis, mean values with standard error of measurement (s.e.m.) were presented in most graphs that were derived from several repeats of biological experiments. Statistical significance was assessed by unpaired two-tailed Student's $t$-test. The Kaplan-Meier method was used to estimate the survival curves for the patient. For animal survival analysis, log-rank (Mantel-Cox) test was used.

\section{ABBREVIATIONS}

MSCs, mesenchymal stromal cells; TGF $\beta$, transforming growth factor- $\beta$; dn-TGFBR2, dominant negative form of TGF $\beta$ receptor 2 .

\section{CONFLICT OF INTEREST}

The authors declare no conflict of interest.

\section{ACKNOWLEDGEMENTS}

This study was supported by grants from the Programs of National Natural Science of China (81330046, 81273316, 81571612, 81530043), the Scientific Innovation Project of the Chinese Academy of Science (XDA 01040100), the Ministry of Science and Technology of China (2015CB964400), the External Cooperation Program of BIC, Chinese Academy of Sciences (GJHZ201307), Shanghai Rising-Star Program (14QA1404200) and the Youth Innovation Promotion Association, Chinese Academy of Sciences.

\section{REFERENCES}

1 Granot Z, Henke E, Comen EA, King TA, Norton L, Benezra R. Tumor entrained neutrophils inhibit seeding in the premetastatic lung. Cancer Cell 2011; 20: 300-314

2 Ohnuki H, Jiang K, Wang D, Salvucci O, Kwak H, Sanchez-Martin D et al. Tumor-infiltrating myeloid cells activate DII4/Notch/TGF-beta signaling to drive malignant progression. Cancer Res 2014; 74: 2038-2049.

3 Shaked Y, Henke E, Roodhart JM, Mancuso P, Langenberg MH, Colleoni M et al. Rapid chemotherapy-induced acute endothelial progenitor cell mobilization: implications for antiangiogenic drugs as chemosensitizing agents. Cancer Cell 2008; 14: 263-273.

4 Ho IA, Chan KY, Ng WH, Guo CM, Hui KM, Cheang P et al. Matrix metalloproteinase 1 is necessary for the migration of human bone marrow-derived mesenchymal stem cells toward human glioma. Stem Cells 2009; 27: 1366-1375.
5 Dwyer RM, Potter-Beirne SM, Harrington KA, Lowery AJ, Hennessy E, Murphy JM et al. Monocyte chemotactic protein-1 secreted by primary breast tumors stimulates migration of mesenchymal stem cells. Clin Cancer Res 2007; 13: 5020-5027.

6 Rattigan Y, Hsu JM, Mishra PJ, Glod J, Banerjee D. Interleukin 6 mediated recruitment of mesenchymal stem cells to the hypoxic tumor milieu. Exp Cell Res 2010; 316: 3417-3424.

7 Frenette PS, Pinho S, Lucas D, Scheiermann C. Mesenchymal stem cell: keystone of the hematopoietic stem cell niche and a stepping-stone for regenerative medicine. Annu Rev Immunol 2013; 31: 285-316.

8 Öhlund D, Elyada E, Tuveson D. Fibroblast heterogeneity in the cancer wound. J Exp Med 2014; 211: 1503-1523.

9 Karnoub AE, Dash AB, Vo AP, Sullivan A, Brooks MW, Bell GW et al. Mesenchymal stem cells within tumour stroma promote breast cancer metastasis. Nature 2007; 449: 557-563.

10 Jung Y, Kim JK, Shiozawa Y, Wang J, Mishra A, Joseph J et al. Recruitment of mesenchymal stem cells into prostate tumours promotes metastasis. Nat Commun 2013; 4: 1795.

11 Giacobbe A, Compagnone M, Bongiornoborbone L, Antonov A, Markert E, Zhou J et al. p63 controls cell migration and invasion by transcriptional regulation of MTSS1. Oncogene 2015; 35: 1602-1608.

12 Memmi E, Sanarico A, Giacobbe A, Peschiaroli A, Frezza V, Cicalese A et al. p63 sustains self-renewal of mammary cancer stem cells through regulation of Sonic Hedgehog signaling. Proc Natl Acad Sci USA 2015; 112: 3499-3504.

13 Melino G, Memmi E, Pelicci P, Bernassola F. Maintaining epithelial stemness with p63. Sci Signal 2015; 8: re9.

14 Orimo A, Gupta PB, Sgroi DC, Arenzana-Seisdedos F, Delaunay T, Naeem R et al. Stromal fibroblasts present in invasive human breast carcinomas promote tumor growth and angiogenesis through elevated SDF-1/CXCL12 secretion. Cell 2005; 121: 335-348.

15 Ren G, Zhao X, Wang Y, Zhang X, Chen X, Xu C et al. CCR2-dependent recruitment of macrophages by tumor-educated mesenchymal stromal cells promotes tumor development and is mimicked by TNFalpha. Cell Stem Cell 2012; 11: 812-824.

16 Grivennikov SI, Greten FR, Karin M. Immunity, inflammation, and cancer. Cell 2010; 140: 883-899.

17 Boimel PJ, Smirnova T, Zhou ZN, Wyckoff J, Park H, Coniglio SJ et al. Contribution of CXCL12 secretion to invasion of breast cancer cells. Breast Cancer Res 2012; 14: R23.

18 Ganju RK, Deol YS, Nasser MW. Role of CXCL12 and CXCR4 in Tumor Biology and Metastasis. In: Fatatis A (ed). Signaling Pathways and Molecular Mediators in Metastasis. Springer Netherlands: Dordrecht, 2012, pp 221-243.

19 Zlotnik A, Burkhardt AM, Homey B. Homeostatic chemokine receptors and organ-specific metastasis. Nat Rev Immunol 2011; 11: 597-606.

20 Smith MC, Luker KE, Garbow JR, Prior JL, Jackson E, Piwnica-Worms D et al. CXCR4 regulates growth of both primary and metastatic breast cancer. Cancer Res 2004; 64: 8604-8612.

21 Wright N, de Lera TL, Garcia-Moruja C, Lillo R, Garcia-Sanchez F, Caruz A et al. Transforming growth factor-beta1 down-regulates expression of chemokine stromal cell-derived factor-1: functional consequences in cell migration and adhesion. Blood 2003; 102: 1978-1984.

22 Pickup M, Novitskiy S, Moses HL. The roles of TGFbeta in the tumour microenvironment. Nat Rev Cancer 2013; 13: 788-799.

23 Xu C, Yu P, Han X, Du L, Gan J, Wang Y et al. TGF-beta promotes immune responses in the presence of mesenchymal stem cells. J Immunol 2014; 192: 103-109.

24 Ikushima H, Miyazono K. TGFbeta signalling: a complex web in cancer progression. Nat Rev Cancer 2010; 10: 415-424.

25 Vanharanta S, Massague J. Origins of metastatic traits. Cancer Cell 2013; 24: 410-421.

26 De Luca A, Lamura L, Gallo M, Maffia V, Normanno N. Mesenchymal stem cellderived interleukin-6 and vascular endothelial growth factor promote breast cancer cell migration. J Cell Biochem 2012; 113: 3363-3370.

27 Wang J, Loberg R, Taichman RS. The pivotal role of CXCL12 (SDF-1)/CXCR4 axis in bone metastasis. Cancer Metastasis Rev 2006; 25: 573-587.

28 Teicher BA, Fricker SP. CXCL12 (SDF-1)/CXCR4 pathway in cancer. Clin Cancer Res 2010; 16: 2927-2931.

29 Burns JM, Summers BC, Wang Y, Melikian A, Berahovich R, Miao Z et al. A novel chemokine receptor for SDF-1 and I-TAC involved in cell survival, cell adhesion, and tumor development. J Exp Med 2006; 203: 2201-2213.

30 Miao Z, Luker KE, Summers BC, Berahovich R, Bhojani MS, Rehemtulla A et al. CXCR7 (RDC1) promotes breast and lung tumor growth in vivo and is expressed on tumor-associated vasculature. Proc Natl Acad Sci USA 2007; 104: 15735-15740.

31 Meijer J, Ogink J, Roos E. Effect of the chemokine receptor CXCR7 on proliferation of carcinoma cells in vitro and in vivo. Br J Cancer 2008; 99: 1493-1501. 
MSCs promote tumour metastasis via regulating CXCL12

PF Yu et al

32 Arabanian LS, Fierro FA, Stolzel F, Heder C, Poitz DM, Strasser RH et al. MicroRNA-23a mediates post-transcriptional regulation of CXCL12 in bone marrow stromal cells. Haematologica 2014; 99: 997-1005.

33 Kim M, Koh YJ, Kim KE, Koh Bl, Nam DH, Alitalo K et al. CXCR4 signaling regulates metastasis of chemoresistant melanoma cells by a lymphatic metastatic niche. Cancer Res 2010; 70: 10411-10421.

34 Ray P, Stacer A, Fenner J, Cavnar S, Meguiar K, Brown M et al. CXCL12-|[gamma]| in primary tumors drives breast cancer metastasis. Oncogene 2015; 34: 2043.

35 Duvallnoelle N, Karwandyar A, Richmond A, Raman D. LASP-1: a nuclear hub for the UHRF1-DNMT1-G9a-Snail1 complex. Oncogene 2015; 35: 1122-1133.

36 Balabanian K, Lagane B, Infantino S, Chow KY, Harriague J, Moepps B et al. The chemokine SDF-1/CXCL12 binds to and signals through the orphan receptor RDC1 in T lymphocytes. J Biol Chem 2005; 280: 35760-35766.
37 Stacer A, Fenner J, Cavnar S, Xiao A, Zhao S, Chang S et al. Endothelial CXCR7 regulates breast cancer metastasis. Oncogene 2015; 35: 1716-1724. NonCommercial-ShareAlike 4.0 International License. The images or other third party material in this article are included in the article's Creative Commons license, unless indicated otherwise in the credit line; if the material is not included under the Creative Commons license, users will need to obtain permission from the license holder to reproduce the material. To view a copy of this license, visit http:// creativecommons.org/licenses/by-nc-sa/4.0/

(c) The Author(s) 2017

Supplementary Information accompanies this paper on the Oncogene website (http://www.nature.com/onc) 